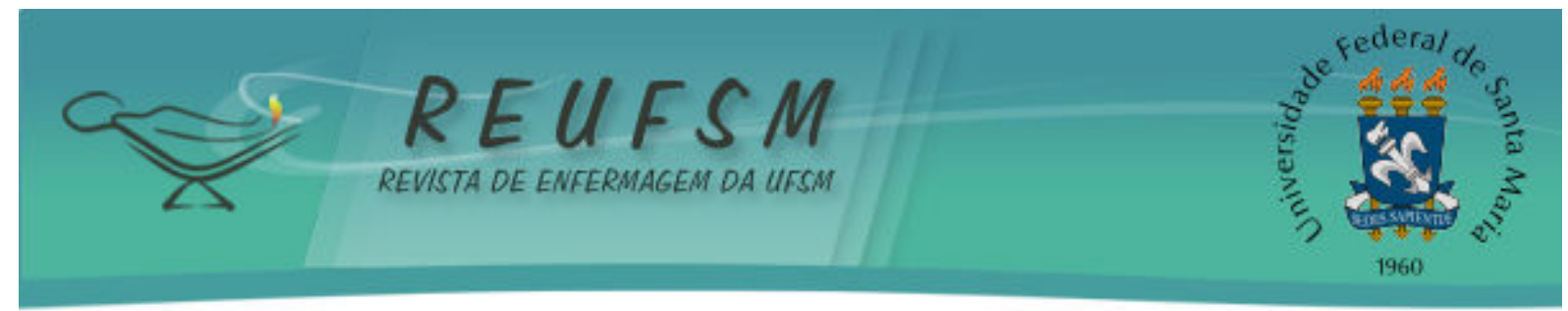

ARTIGO ORIGINAL

\title{
CAPACITAÇÃO DE ENFERMEIROS DE UM HOSPITAL UNIVERSITÁRIO PÚBLICO NA GESTÃO DE CUSTO
}

\section{TRAINING OF NURSES OF A PUBLIC UNIVERSITY HOSPITAL ON COST MANAGEMENT FORMACIÓN DE ENFERMEROS DE UN HOSPITAL UNIVERSITARIO PÚBLICO EN GESTIÓN DE COSTES}

Doi: $10.5902 / 2179769212938$

\author{
William Tiago de Oliveira ${ }^{1}$ \\ Maria do Carmo Lourenço Haddad² \\ Marli Terezinha Oliveira Vannuchi ${ }^{3}$ \\ Ana Vanessa Deffácio Rodrigues ${ }^{4}$ \\ Paloma de Souza Cavalcante Pissinati ${ }^{5}$
}

RESUMO: Objetivo: descrever a elaboração, aplicação e avaliação de um curso de capacitação em gestão de custo para enfermeiros de um hospital universitário público. Método: trata-se de um estudo exploratório-descritivo, de natureza qualitativa. Utilizou-se a técnica de grupo focal como forma de promover a aproximação, integração e envolvimento dos participantes e como instrumento de avaliação, sendo realizada uma sessão antes da capacitação e imediatamente após. 0 curso foi ministrado em novembro de 2010, com carga horária de três horas-aulas e teve como estratégia de aprendizagem aulas expositiva dialogadas. Resultados: a capacitação buscou ampliar a visão dos enfermeiros sobre o gerenciamento de custo, bem como instigar a reflexão sobre a importância estratégica destes profissionais nas organizações hospitalares. Conclusão: o curso promoveu a sensibilização dos enfermeiros sobre a importância da gestão de custo, propiciando a estes profissionais novos conhecimentos. Contudo, é preciso aprofundar as discussões sobre a temática apresentada.

Descritores: Enfermeiros; Hospitais universitários; Custos hospitalares.

ABSTRACT: Objective: to describe the design, implementation and evaluation of a training course on cost management for nurses in a public university hospital. Method: this is an exploratory-descriptive qualitative study. The focus group technique was utilized as a way to promote creation of rapport, integration and involvement of participants, as well as an evaluation tool, in which a session was held before the training and another one immediately after it. The course was taught in November 2010 with a workload of three hours and used dialogic expository lessons as a learning strategy. Results: the training aimed to improve nurses' views on cost management, as well as to prompt reflections about the strategic importance of these professionals in hospitals. Conclusion: the course promoted the awareness of the nurses about the importance of cost management, providing these professionals with new knowledge about it. However, it is necessary to deepen discussions on the theme.

\footnotetext{
${ }^{1}$ Enfermeiro. Mestre em Enfermagem. Docente do Departamento de Enfermagem da Faculdade Ingá, Maringá, Paraná, Brasil. E-mail: oliveirawt@hotmail.com

${ }^{2}$ Enfermeira. Professora Doutora do Departamento de Enfermagem da Universidade Estadual de Londrina, Londrina, Paraná, Brasil. E-mail: carmohaddad@gmail.com

${ }^{3}$ Enfermeira. Professora Doutora do Departamento de Enfermagem da Universidade Estadual de Londrina, Londrina, Paraná, Brasil. E-mail: vannuchi@sercomtel.com.br

${ }^{4}$ Enfermeira. Mestre em Enfermagem pela Universidade Estadual de Maringá, Londrina, Paraná, Brasil. E-mail: vanessa_deffaccio@hotmail.com

${ }^{5}$ Enfermeira. Mestranda do Programa de Pós Graduação em Enfermagem da Universidade Estadual de Londrina, Londrina, Paraná, Brasil. E-mail: cavalcanteps7@gmail.com
} 


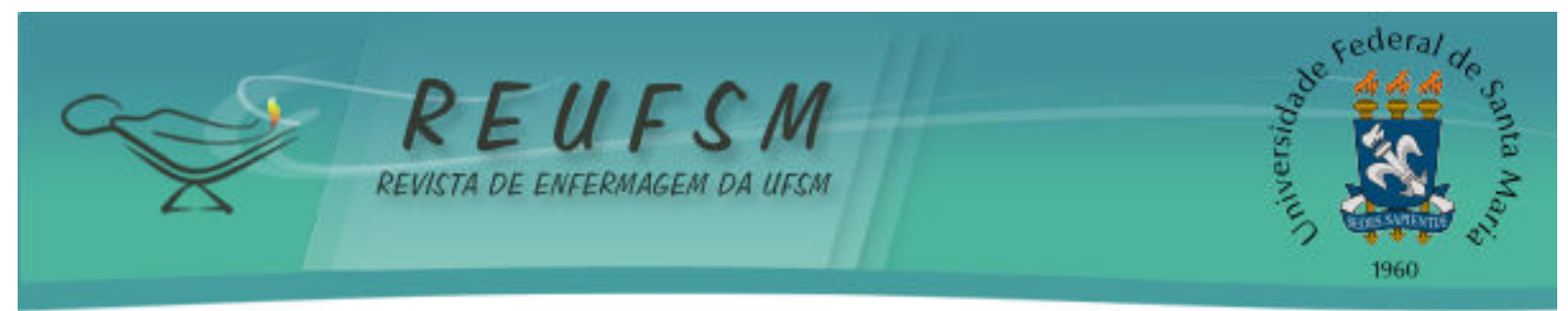

Descriptors: Nurses, male; Hospitals, university; Hospitals costs.

RESUMEN: Objetivo: describir el diseño, implementación y evaluación de um curso de formación en gestión costes para los enfermeros de un hospital universitario. Método: estudio exploratorio-descriptivo cualitativo. Se utilizó la técnica del grupo focal como forma de promover la aproximación, integración y participación de los participantes y como herramienta de evaluación, realizando una reunión antes de la formación $e$ inmediatamente después. El curso fue efectuado en noviembre de 2010 con un horario de tres horas por clase y tuvo como estrategia de aprendizaje la exposición de lecciones. Resultados: la formación buscó ampliar la perspectiva de los enfermeros sobre gestión de costes, así como instigar a la reflexión sobre la importancia estratégica de estos profesionales en los hospitales. Conclusión: el curso promovió la sensibilización de los enfermeros sobre la importancia de la gestión de costes, ofreciendo nuevos conocimientos a estos profesionales. No obstante, es necesario profundizar en las discusiones sobre el tema.

Descriptores: Enfermeros; Hospitales universitarios; Costos de hospital.

\section{INTRODUÇÃO}

A abordagem sobre gestão de custo na área da saúde vem ganhando projeção nos últimos anos em decorrência dos constantes aumentos de gastos neste setor. Fatores relacionados a competitividade do mercado, a pressão da sociedade e dos planos de saúde, e o controle de preço do governo têm feito com que os hospitais adotem o gerenciamento de custo como estratégia de trabalho visando a obtenção de informações importantes no monitoramento dos recursos. ${ }^{1}$

Esta nova perspectiva tem sido considerada pelos gestores dos hospitais como uma ferramenta essencial no processo administrativo, permitindo identificar caminhos estratégicos mais lucrativos e proporcionando a análise dos gastos desnecessários que assim poderão ser eliminados ou diminuídos. ${ }^{2}$

Atualmente, verifica-se que o enfermeiro vem ganhando cada vez mais espaço enquanto gestor dos custos nos serviços de saúde, sendo apontado pela Organização Mundial da Saúde como aquele com maior potencial na área da saúde para assegurar uma assistência rentável, ou seja, eficaz em função dos custos. ${ }^{3}$

Ao enfermeiro cabe a responsabilidade pelo gerenciamento de recursos humanos, materiais e financeiros, muitas vezes escassos. Neste contexto, torna-se necessária a aquisição de conhecimentos por parte deste profissional sobre a temática do gerenciamento de custos. Isto reforça a importância da inserção desses conteúdos nos cursos de graduação e pós-graduação de enfermagem, a fim de prepararem os futuros profissionais para gerenciarem os custos da assistência de enfermagem. ${ }^{2}$

Tendo em vista os desafios postos pelo panorama atual, e com o objetivo de oportunizar a qualificação dos enfermeiros como gestor de custo, surgiu a proposta de promover a capacitação destes profissionais sobre a problemática apresentada.

A ideia de elaborar um curso em gestão de custo surgiu através das evidências obtidas na pesquisa apresentada no Congresso de Administração Hospitalar (ADH'2010), em São Paulo: "Concepções de enfermeiros de um Hospital Universitário Público sobre o relatório gerencial de custo". Nesse estudo, ${ }^{4}$ foi diagnosticada a necessidade de promover a capacitação dos enfermeiros na prática do gerenciamento de custo.

Portanto, o objetivo deste estudo foi descrever a elaboração, aplicação e avaliação de um curso de capacitação em gestão de custo para enfermeiros de um hospital universitário público. 


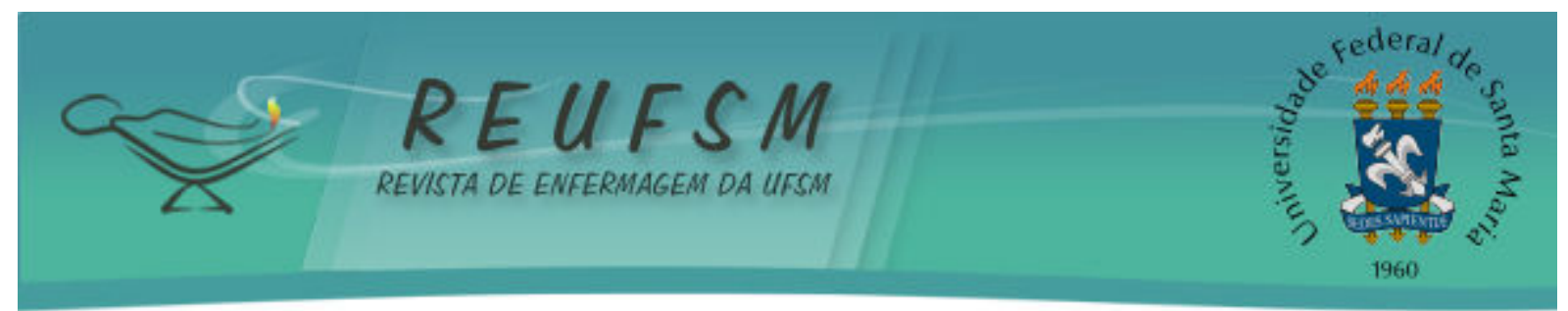

Espera-se que este estudo possa contribuir com as instituições de saúde no sentido de destacar o papel do enfermeiro enquanto gestor de custo, bem como, orientar as ações deste profissional, através da fundamentação teórica, assumindo a responsabilidade perante a equipe de enfermagem no sentido de racionalizar 0 uso de materiais $e$ equipamentos nas instituições hospitalares, uma vez que trata-se de um tema novo e de profunda relevância em sua prática profissional.

\section{MÉTODO}

Trata-se de um estudo de natureza qualitativa, descritiva e exploratória. Os dados da pesquisa constituem-se na descrição do planejamento, aplicação e avaliação de uma capacitação em gestão de custo para enfermeiros de um hospital universitário público ministrado no dia 25 de novembro de 2010.

O público alvo desse curso foi composto pela totalidade dos enfermeiros lotados na diretoria de enfermagem da instituição em estudo que atuavam em cargos de chefia, totalizando 12 enfermeiros, sendo um o diretor de enfermagem, três enfermeiros assessores e oito enfermeiros que ocupavam cargo de chefe de divisão.

Optou-se pela utilização da técnica do grupo focal como forma de promover a aproximação, integração e envolvimento dos participantes da pesquisa. 0 processo de avaliação do curso também foi realizado no grupo focal, através das análises dos discursos dos participantes da pesquisa.

Como técnica da pesquisa qualitativa, o grupo focal tem sido utilizado como instrumento de avaliação de programa educacional em saúde. Mostra-se adequado para auxiliar no processo de trabalho de enfermagem, dada a possibilidade de avaliar tanto as potencialidades quanto as fragilidades internas, além de refletir sobre oportunidades e desafios externos que requerem adequações institucionais. ${ }^{5}$

Portanto foram planejadas, estrategicamente, duas sessões de grupo focal: uma que precedeu o curso, visando a compreensão das experiências prévias dos participantes da pesquisa sobre o tema abordado e outra imediatamente após a capacitação com o objetivo de avaliar o curso em si.

Para promover o debate sobre o tema, foram utilizados os seguintes questionamentos: Como você qualifica as questões envolvendo gestão de custo na enfermagem? Quais as dificuldades encontradas no desenvolvimento de ações em gestão de custo?

Para avaliar o curso, as perguntas norteadoras foram: Qual a sua avaliação sobre este curso? Qual a contribuição deste curso para você enquanto enfermeiro?

Para coleta de dados da investigação no grupo focal, utilizou-se o recurso de gravação em áudio, visando garantir a transcrição fidedigna dos dados e possibilitar a compreensão destes pela temática pesquisada.

Após a coleta, as falas dos sujeitos foram transcritas e lidas de modo exaustivo e repetidamente, a fim de discriminar as "unidades de significado" e construir as categorias empíricas. ${ }^{6}$ Finalmente, foi realizada a discussão final que relacionou os dados encontrados aos referenciais literários acerca da temática pesquisada.

As unidades de significados foram identificadas com a letra "S", seguidas por numeração conforme a ordem das entrevistas, de forma a preservar a identidade dos sujeitos.

A pesquisa foi realizada de forma a garantir o cumprimento dos preceitos éticos da Resolução 196/96 sobre pesquisa envolvendo seres humanos, ${ }^{7}$ precedida pelo encaminhamento do projeto de pesquisa ao Comitê de Ética em Pesquisa da Universidade Estadual de Londrina, tendo obtido parecer favorável com o ${ }^{\circ} 140 / 09$, conforme Certificado de Apresentação para Apreciação Ética (CAAE) $\mathrm{n}^{\circ}$ 0117.0.268.268-09. Obteve-se a autorização institucional para a execução da coleta de dados e a assinatura do termo de consentimento livre e esclarecido dos sujeitos da pesquisa. 


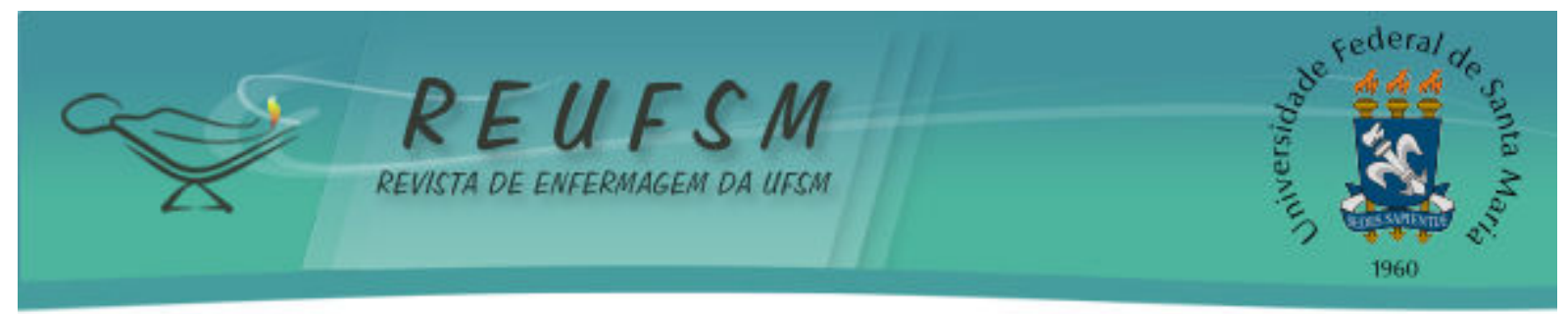

RESULTADOS E DISCUSSÃO

Os resultados deste estudo são apresentados e discutidos em duas categorias.

\section{Elaboração e aplicação da capacitação}

Para a elaboração do curso, formou-se um grupo de trabalho constituído por uma equipe multiprofissional composta por enfermeiros e contabilistas. A formação deste grupo teve como objetivo direcionar as atividades de elaboração e aplicação do curso, a fim de demonstrar aos participantes a sua importância na racionalização e otimização dos recursos disponíveis, bem como, atender as necessidades da instituição em estudo de maneira global.

As primeiras reuniões realizadas entre os integrantes desse grupo tiveram como objetivo analisar os resultados da pesquisa apresentada no ADH'20104, com o intuito de desvelar a percepção dos enfermeiros sobre a problemática em questão. A partir das análises desses resultados, foram identificados os principais aspectos a serem considerados como debilidades em relação à prática do gerenciamento de custo pelo enfermeiro no âmbito hospitalar, sendo apontados: a abordagem sobre gestão de custo como um tema recente dentro do campo da enfermagem; os conhecimentos em gerenciamento de custo não fizeram parte da formação profissional dos enfermeiros; falta de tempo dos enfermeiros para o desenvolvimento das questões relacionadas ao gerenciamento das unidades; foco do trabalho dos enfermeiros voltado às questões assistenciais; dificuldades na interpretação dos dados contidos no relatório gerencial de custo; necessidade de capacitação para a utilização do relatório gerencial de custo.

A partir das principais debilidades apontadas pelo grupo, foram traçados os objetivos do curso: fornecer aos enfermeiros noções gerais sobre o gerenciamento de custo hospitalar; apresentar e discutir os processos de gestão de custo do hospital em estudo e capacitar os enfermeiros na análise gerencial do relatório de custo da unidade sob sua responsabilidade.

Definiu-se que a capacitação iria abranger todos os enfermeiros lotados na diretoria de enfermagem da instituição em estudo que ocupavam cargo de chefia, conforme descrito na metodologia. A escolha deste grupo deu-se pela importância estratégica destes profissionais enquanto cargo de chefia e também pelo fato de que os resultados encontrados no estudo apresentado no $\mathrm{ADH}^{\prime} 2010^{4}$ demonstraram serem esses os profissionais que possuem maior conhecimento sobre a temática em questão. Sendo assim, e considerando que a abordagem sobre gestão de custo trata-se de um tema recente dentro do campo da enfermagem e que o objetivo, posteriormente, é o de estender este curso a todos os enfermeiros do hospital, a opção pelos enfermeiros que ocupam cargo de chefia teve como proposta que estes fossem os peritos do curso, e por meio das sugestões feitas por estes, adequar/aprimorar o curso para a posterior aplicação aos demais enfermeiros do hospital.

Num primeiro momento, o curso foi sistematizado para ser ministrado por cinco dias, totalizando 15 horas-aulas. Contudo devido à dificuldade dos enfermeiros em relação a horários para o agendamento do curso, foi definido junto com a direção de enfermagem que o curso teria duração de três horas aulas. Desta forma, procurou-se abranger a essência de cada conteúdo, de modo a transmitir o necessário para o entendimento do assunto, fornecendo subsídios para que os enfermeiros aprofundassem seus conhecimentos posteriormente.

O conteúdo programático foi elaborado de forma a contemplar todos os objetivos do curso, assim estruturado: contabilidade de custos em hospitais (objetivos, benefícios, conceitos básicos e classificação); o custo como ferramenta gerencial (centros de custos); relatório gerencial de custo (apresentação e utilização do relatório).

A divulgação do curso foi realizada por meio de cartas convites informando aos participantes sobre a data, local e horário do curso. 


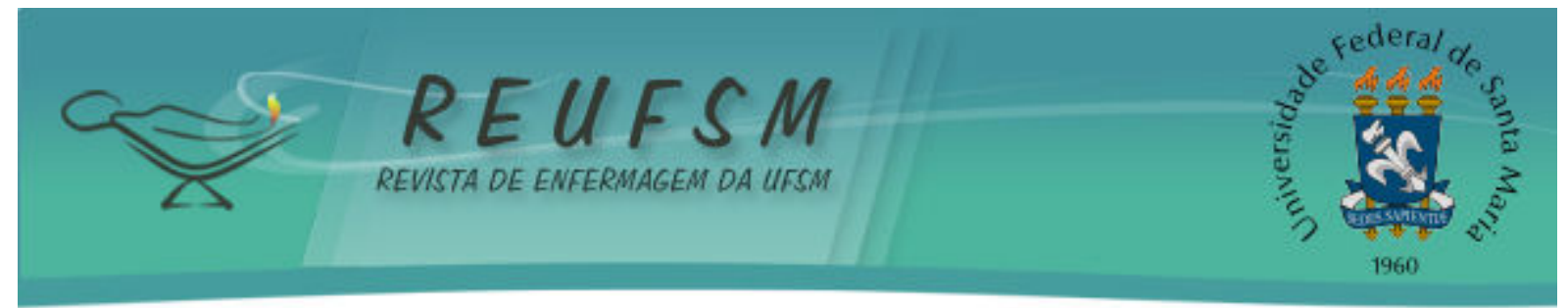

A capacitação foi realizada no período vespertino, durante a jornada de trabalho dos enfermeiros, em local adequado e fora do ambiente de trabalho. A distribuição dos participantes na sala foi estrategicamente preparada de modo a estabelecer um ambiente propício às interações, promovendo o contato visual entre estes. As cadeiras foram dispostas em círculo em número suficiente para todos. 0 material pedagógico utilizado na capacitação foi distribuído nas carteiras, previamente ao início do curso, com o objetivo de que os participantes pudessem acompanhar o conteúdo exposto e fizessem anotações se assim o desejassem.

0 curso foi dividido em duas etapas com intervalo de 15 minutos entre elas. Utilizouse como estratégia de aprendizagem aulas expositiva dialogadas com recursos audiovisuais. A aula expositiva dialogada permite a exposição do conteúdo, com a participação ativa dos estudantes, cujo conhecimento prévio deve ser considerado e pode ser tomado como ponto de partida. O professor leva os estudantes a questionarem, interpretarem e discutirem o objeto de estudo, a partir do reconhecimento e do confronto com a realidade. ${ }^{8}$

Foram apresentados aos enfermeiros os resultados do ADH'2010, ${ }^{4}$ a fim de contextualizar e justificar a necessidade de capacitação dos enfermeiros e com o intuito de sensibilizar os participantes sobre a problemática em questão. Na sequência foi utilizada a técnica do grupo focal conforme descrita na metodologia.

A capacitação foi ministrada por dois autores deste estudo. Seguindo o cronograma apresentado na Figura 1.

\begin{tabular}{c|l}
\hline Horário & \multicolumn{1}{c}{ Conteúdo } \\
\hline 14h às 14h05min & Abertura \\
14h05min às $14 \mathrm{~h} 20 \mathrm{~min}$ & Apresentação dos resultados do ADH'2010 \\
14h20min às $15 \mathrm{~h}$ & Grupo Focal \\
15h às $15 \mathrm{~h} 40 \mathrm{~min}$ & Aula expositivo-dialogada \\
15h40min às $16 \mathrm{~h}$ & Intervalo \\
16h às 16h30min & Aula expositivo-dialogada \\
16h30min às 17h & Avaliação \\
\hline
\end{tabular}

Figura 1 - Cronograma do curso Gestão de Custo ministrado para enfermeiros de um hospital universitário público. Londrina-PR, 2010.

A avaliação do curso deu-se nos minutos finais e foi solicitado que cada participante verbalizasse sua opinião, expondo o que apreenderam.

\section{Avaliação da capacitação}

Evidenciou-se que a avaliação dos enfermeiros referente a capacitação foi positiva. Para eles, essa temática deve ser estendida a todos os servidores do hospital, a fim de que estes compreendam a importância do tema e modifiquem sua postura profissional de forma a minimizar o desperdício dos recursos.

Todos os funcionários precisam ter a conscientização sobre o gerenciamento de custos, em todos os níveis e nas diferentes categorias, e cabe a nós enfermeiros cobrarmos da nossa equipe. (S.1)

Este curso deve ser ampliado para todos os servidores do hospital, com os mesmos enfoques. (S.8) 


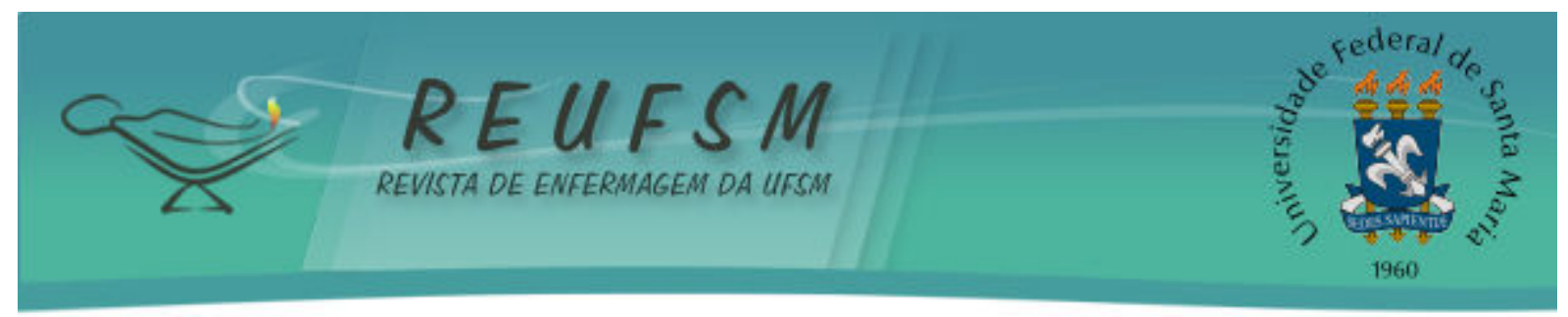

Acho que pode ser estendido para todos os servidores do hospital, nas diferentes categorias profissionais. (S.12)

Os participantes concordaram que o curso ofereceu informações importantes e úteis à prática da gestão de custo, contudo, por se tratar de um tema novo dentro do campo da enfermagem, é preciso aprofundar-se sobre o assunto.

Acho que esse tema é de extrema importância. Contudo penso que é preciso aprofundar mais as discussões a este respeito, pois tratase de um tema novo para nós enfermeiros. (S.6)

A capacitação por meio da educação continuada constitui importante ferramenta para complementar a formação dos profissionais e trata-se de um meio para que estes aprimorem conhecimentos. ${ }^{9}$ Assim, a capacitação sobre custos mostra-se essencial para a compreensão dos trabalhadores e constitui fator relevante para a melhoria da qualidade dos serviços.

Os enfermeiros foram capacitados na utilização do relatório gerencial de custo, o que possibilitou a compreensão e interpretação dos dados, bem como conhecimentos específicos sobre a temática.

Enquanto enfermeiros, precisamos conhecer essas informações sobre a contabilidade de custos do hospital e saber trabalhá-las, a fim de proporcionar um melhor atendimento, com o menor custo. (S.8)

Foi muito bom participar desse curso, dentre outras coisas, aprendi novas terminologias que até então não eram do meu conhecimento... Essas informações serão úteis para sabermos onde devemos economizar. (S.3)

Destaca-se a necessidade dos enfermeiros adquirirem conhecimentos sobre gestão de custo, a fim de que reconheçam seu papel como agentes de mudanças. ${ }^{2}$ Faz-se notório à conscientização e participação deste profissional no sentido de racionalizar os gastos dentro de um serviço de saúde, utilizando o mínimo de recursos possíveis junto com a manutenção da qualidade dos serviços prestados. Sendo assim, o gerenciamento de custos apresenta-se como um processo administrativo que visa conhecer o custo em cada instância, tornando-se um diferencial na tomada de decisão dos enfermeiros e propiciando a este profissional um papel efetivo na racionalização de recursos, controle de desperdícios e otimização de resultados.

Nas falas, observou-se que existe uma diferença cultural marcante entre organizações públicas e privadas quanto ao gerenciamento de custo, de modo que o primeiro poderia ser mais envolvido no controle dos recursos disponíveis.

Nos hospitais particulares existe um controle maior referente aos gastos e no público não, então percebe-se que é cultural. (S.1)

A enfermagem não está avaliando o quanto está sendo gasto a mais, não existe uma preocupação com custo, o que vem de encontro com a cultura de uma instituição pública, contaminou pega outra. (S.7)

Ao discutir a apuração e gestão de custos no setor público, percebe-se uma vasta gama de desafios que necessitam ser superados. Entre os principais desafios estão a dificuldade de criar uma cultura de custos em instituições públicas. Observa-se a necessidade 


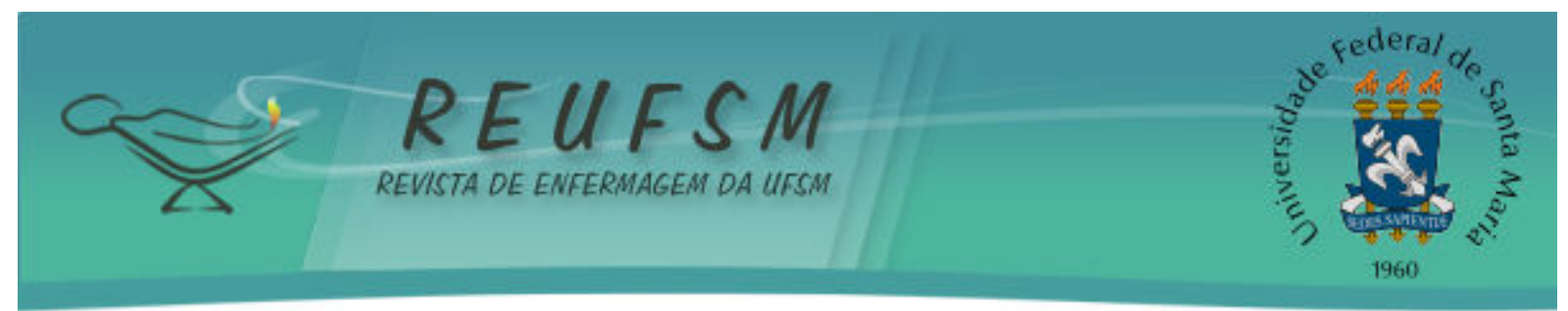

de mudança de paradigma, uma vez que existe à crença de ser este um serviço onde se pode gastar de maneira demasiada, pois não se tem uma prática de controle das ações.

0 desinteresse nas questões referentes à gestão de custo em hospitais públicos pode estar relacionado à ausência de cobranças por parte dos gestores e de uma política voltada para o gerenciamento de custo. Em 2006, o Ministério da Saúde lançou o Programa Nacional de Gestão de Custos, ${ }^{10}$ contudo percebe-se que este programa ainda busca a sistematização, para promover o desenvolvimento de ações que contribuam com a apuração e o controle de custos em saúde.

Como o objetivo do serviço público não é a obtenção de lucros, a busca pelo controle dos custos deveria estar mais voltada para uma melhor aplicação dos recursos, o que iria promover uma distribuição mais eficiente dos gastos públicos. ${ }^{11}$ Deste modo, há necessidade de se gerar dados confiáveis e corretos para a apuração de custos, bem como de profissionais especializados tanto no processo de apuração, quanto na análise dessas informações.

A descontinuidade administrativa é outra situação que pode dificultar a gestão de custo nas organizações públicas, uma vez que a rotatividade dos cargos faz com que os projetos sejam realizados em curto prazo, de modo que estes possam ser concluídos no próprio mandato, gerando descontinuidade dos avanços conquistados pela chefia que foi substituída.

Evidenciou-se, também, que existe uma preocupação no intuito de prepararem os futuros profissionais das diversas áreas de atuação a aplicarem ações de controle dos custos, de modo que estes possam gerir os recursos da melhor forma possível, evitando desperdício.

Os alunos precisam ser envolvidos nessa política de custo e nós temos que mostrar para eles a maneira correta de se comportar. Nós temos que trazê-los para essa realidade, aqui funciona assim e você vai se comportar desta forma. (S.11)

A desculpa é os alunos, contudo nós temos a responsabilidade de instruir não só os alunos como também os servidores. (S.2)

A maioria dos hospitais universitários é mantida por verbas públicas e são caracterizados, primeiramente, como centros de atendimento de alta complexidade, sendo referência na região onde atuam. Nesses hospitais, além da prestação de serviços especializados com forte exigência tecnológica, incorporam-se as atividades de ensino e pesquisa, o que contribui para o maior custo quando comparados aos hospitais não universitários. ${ }^{12}$

Os hospitais de ensino devem primar pela eficiência dos serviços prestados à sociedade, pois além da assistência a saúde, estes tem a importante missão de formação de recursos humanos. ${ }^{13}$ Neste sentido, é importante que se pratiquem ações voltadas à prática da gestão de custo, a fim de proporcionarem aos futuros profissionais uma reflexão, para que se tornem conscientes e contribuam para redução dos custos. Para tanto, é preciso que os hospitais estejam estruturados para possibilitar a apuração dos custos com precisão e proporcionar informações para a tomada de decisão.

Alguns enfermeiros referiram utilizar as informações sobre custos para sensibilizar os funcionários sobre os elevados gastos de uma internação e a importância do uso correto de materiais e equipamentos.

À medida que se passa a conhecer os gastos, os custos dos materiais, podemos passar para os nossos funcionários o custo daquele produto e cobrar desse funcionário maior controle dos gastos. (S.7)

É necessário, que o enfermeiro líder da equipe de enfermagem utilize estes conhecimentos adquiridos com o intuito de promover a qualidade, eficácia e eficiência da 


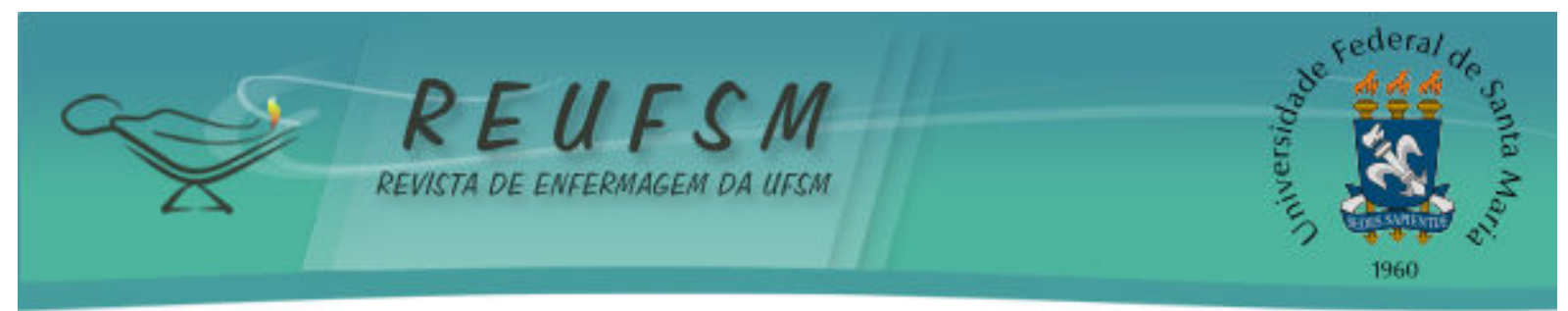

assistência de enfermagem. ${ }^{14}$ Destarte, cabe ao enfermeiro o compromisso de incorporar o gerenciamento de custos em sua prática profissional, identificando o custo de cada atividade e cobrar dos servidores maior empenho no sentido de otimizar os recursos disponíveis.

\section{CONSIDERAÇÕES FINAIS}

A capacitação dos enfermeiros na gestão de custo hospitalar teve como objetivo preparar esses profissionais a atuarem de forma a racionalizar os gastos dentro do serviço hospitalar, utilizando o mínimo de recursos possíveis com hábitos e atitudes condizentes às exigências do mercado, de forma a promover a manutenção da qualidade dos serviços prestados.

A partir das discussões em grupo, percebeu-se a importância de capacitar os enfermeiros na gestão de custo, pois se trata de um tema recente dentro do campo da enfermagem e por considerar que este profissional ocupa um papel de destaque para o equilíbrio financeiro das instituições hospitalares.

Evidenciou-se a necessidade de mudança na cultura dos servidores das instituições hospitalares públicas, uma vez que estes não estão acostumados a prestar contas das ações desenvolvidas, sendo necessário que existam cobranças por parte dos gestores e de uma política voltada para o gerenciamento de custo.

Cabe aos trabalhadores dos hospitais de ensino incentivar os futuros profissionais a gerenciarem custos em sua prática. Assim, é preciso que a instituição possibilite a apuração dos custos e forneça informações que direcionem as tomadas de decisões.

Na percepção dos enfermeiros, a capacitação em gestão de custo deve ser expandida a todos os servidores do hospital, pois todos são responsáveis pelo uso racional dos recursos gastos na assistência e precisam estar capacitados para o gerenciamento de custos, de modo que o sucesso no gerenciamento deste depende da conscientização de todas as áreas envolvidas.

Conclui-se que como primeira abordagem o curso serviu para sensibilizar os enfermeiros sobre a importância da gestão de custo, propiciando a estes profissionais novos conhecimentos. Contudo, é preciso aprofundar-se nas discussões sobre a temática apresentada, tornando-se necessária a busca de mecanismos para estimular a participação dos enfermeiros nesse processo.

\section{REFERÊNCIAS}

1. Dallora MELV, Forster AC. A importância da gestão de custos em hospitais de ensino considerações teóricas. Medicina (Ribeirão Preto) [Internet]. 2008 abr/jun [acesso em 2014 fev 26];41(2):135-42. Disponível em: http://revista.fmrp.usp.br/2008/VOL41N2/rev_a_importancia_gestao_custos_hospitais_en sino.pdf.

2. Oliveira WT, Rodrigues AVD, Haddad MCL, Vannuchi MTO, Taldivo MA. Concepções de enfermeiros de um hospital universitário público sobre o relatório gerencial de custos. Rev Esc Enferm USP [Internet]. 2012 out [acesso em 2014 fev 26];46(5):1184-91. Disponível em: http://www.scielo.br/pdf/reeusp/v46n5/21.pdf.

3. Consejo Internacional de Enfermeras. La calidad, los costos y la enfermería. Ginebra: CIE; 1993.

4. Oliveira WT, Rodrigues AVD, Haddad MCL, Vannuchi MTO, Taldivo MA. Concepções de enfermeiros de um hospital universitário público sobre o relatório gerencial de custo. In: Anais XI Congresso Brasileiro de Qualidade em Enfermagem; 2010 maio 26-27; São Paulo. 


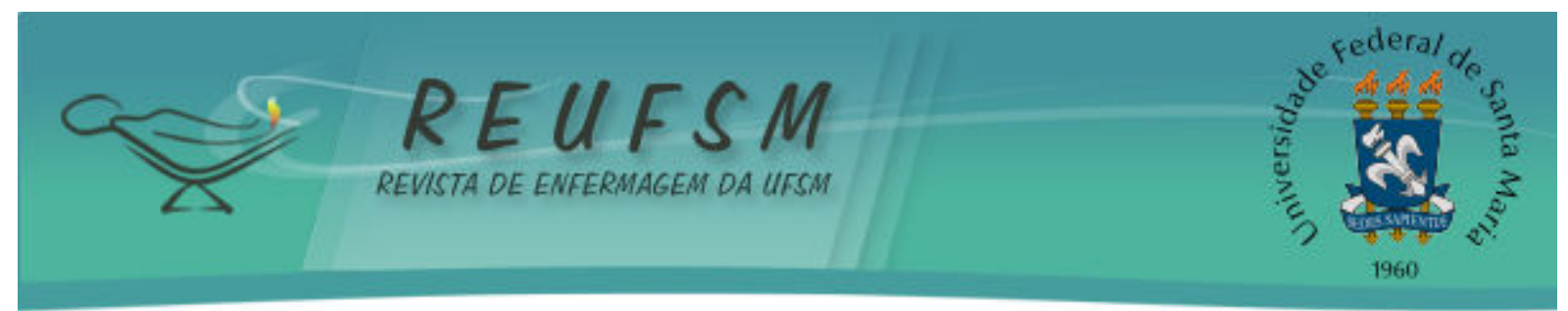

5. Backes DS, Colomé JS, Erdmann RH, Lunardi VL. Grupo focal como técnica de coletas e análise de dados em pesquisas qualitativas. Mundo Saúde. 2011;35(4):438-42.

6. Josgrilberg RS. O método fenomenológico e as ciências humanas. In: Castro DSP, organizador. Fenomenologia e análise do existir. São Paulo: Umesp, Sobraphe; 2000. p. 7593.

7. Brasil. Ministério da Saúde. Conselho Nacional de Saúde. Resolução CNS n 196, de 10 de outubro de 1996. Aprova diretrizes e normas regulamentadoras de pesquisas envolvendo seres humanos. Bioética (Brasília). 1996;4(2 Supl):15-25.

8. Anastasiou LGC, Alves LP. Processos de ensinagem na universidade: pressupostos para as estratégias de trabalho em aula. Joinville (SC): Univille; 2003.

9. Lazzari DD, Schmidt N, Jung W. Educação continuada em unidade de terapia intensiva na percepção de enfermeiras. Rev Enferm UFSM [Internet]. 2012 jan/abr [acesso em 2014 fev 26];2(1):88-96.Disponível em: http://cascavel.cpd.ufsm.br/revistas/ojs2.2.2/index.php/reufsm/article/view/4592/3130.

10. Brasil. Ministério da Saúde. Secretaria de Ciência, Tecnologia e Insumos Estratégicos. Departamento de Economia e Saúde. Programa Nacional de Gestão de Custos: manual técnico de custos - conceitos e metodologias. Brasília: Ministério da Saúde; 2006. (Série A. Normas e manuais técnicos).

11. Almeida AG, Borba JA, Flores LCS. A utilização das informações de custos na gestão da saúde pública: um estudo preliminar em secretarias municipais de saúde do estado de Santa Catarina. Rev Adm Pública [Internet]. 2009 jun [acesso em 2014 fev 26];43(3):579607. Disponível em: http://www.scielo.br/scielo.php?pid=S003476122009000300004 \&script=sci_arttext.

12. Bonacim CAG, Araujo AMP. Gestão de custos aplicada a hospitais universitários públicos: a experiência do hospital das clínicas da faculdade de medicina de Ribeirão Preto da USP. Rev Adm Pública [Internet]. 2010 ago [acesso em $2014 \mathrm{fev}$ 26];44(4):903-31. Disponível em: http://www.scielo.br/scielo.php?script=sci_arttext\&pid=S003476122010000400007.

13. Barros CC, Silva JDG, Souza FJV, Melo MMD, Taveira LB. Há sistemas de custos nos hospitais universitários federais? Rev Estudos Contábeis [Internet]. 2013 jan/jun [acesso em 2014 fev 26];4(6):78-98. Disponível em: http://www.uel.br/revistas/uel/index.php/rec/article/view/14262/12605.

14. Costa DB, Vannuchi MTO, Haddad MCFL, Cardoso MGP, Silva LG, Garcia SD. Custo de educação continuada para equipe de enfermagem de um hospital universitário público. Rev Eletrônica Enferm [Internet]. 2012 abr/jun [acesso em 2014 fev 26];14(2):257-66. Disponível em: http://www.fen.ufg.br/revista/v14/n2/v14n2a05.htm.

Data de recebimento: 17/02/2014

Data de aceite: 15/09/2014

Contato com autor responsável: William Tiago de Oliveira

Endereço: Travessa Mandaguari, 106, Bairro Centro, Marialva-PR. CEP: 86990-000.

Email: oliveirawt@hotmail.com 